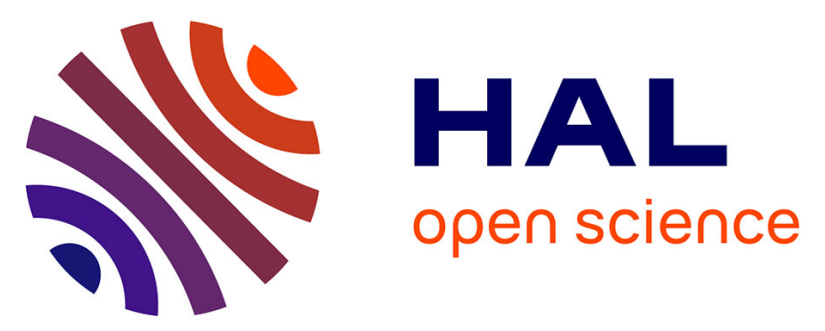

\title{
Application of the Direct Quadrature Method of Moments for the modelling of the enzymatic hydrolysis of cellulose: II. Case of insoluble substrate
}

Noureddine Lebaz, Arnaud Cockx, Mathieu Sperandio, Alain Liné, Jérôme Morchain

\section{To cite this version:}

Noureddine Lebaz, Arnaud Cockx, Mathieu Sperandio, Alain Liné, Jérôme Morchain. Application of the Direct Quadrature Method of Moments for the modelling of the enzymatic hydrolysis of cellulose: II. Case of insoluble substrate. Chemical Engineering Science, 2016, 149, pp.322 - 333. 10.1016/j.ces.2016.04.029 . hal-01876392

\section{HAL Id: hal-01876392 https://hal.science/hal-01876392}

Submitted on 16 Nov 2020

HAL is a multi-disciplinary open access archive for the deposit and dissemination of scientific research documents, whether they are published or not. The documents may come from teaching and research institutions in France or abroad, or from public or private research centers.
L'archive ouverte pluridisciplinaire HAL, est destinée au dépôt et à la diffusion de documents scientifiques de niveau recherche, publiés ou non, émanant des établissements d'enseignement et de recherche français ou étrangers, des laboratoires publics ou privés. 


\title{
Application of the Direct Quadrature Method of Moments for the modelling of the enzymatic hydrolysis of cellulose : II. Case of insoluble substrate
}

\author{
Noureddine Lebaz ${ }^{\mathrm{a}, \mathrm{b}, \mathrm{c}, \mathrm{d}}$, Arnaud Cockx ${ }^{\mathrm{b}, \mathrm{c}, \mathrm{d}}$, Mathieu Spérandio ${ }^{\mathrm{b}, \mathrm{c}, \mathrm{d}}$, Alain Linéb,c,d, Jérôme Morchain ${ }^{\mathrm{b}, \mathrm{c}, \mathrm{d}, *}$ \\ ${ }^{a}$ Toulouse White Biotechnology (UMS INRA/INSA/CNRS), 3 rue Ariane, 31520 Ramonville Saint Agne, France \\ ${ }^{b}$ Université de Toulouse; INSA, UPS, INP; LISBP, 135 Avenue de Rangueil, F-31077, Toulouse, France \\ ${ }^{c}$ INRA, UMR792 Ingénierie des Systèmes Biologiques et des Procédés, F-31400, Toulouse, France \\ ${ }^{d}$ CNRS, UMR5504, F-31400, Toulouse, France
}

\begin{abstract}
In the second part of this study, the population balance model developed for the enzymatic hydrolysis of cellulose polymer chains is extended to particulate cellulose substrates. In this case, the particle fragmentation process is complex. This phenomenon is induced by the hydrodynamic shear stress when the particle cohesion is lower than the hydrodynamic force. The particle cohesion depends on the mechanical properties of the substrate particles which are affected by the Endoglucanase (EG) activity that hydrolyses accessible glycosidic bonds all along the reaction. These two effects are taken into account in the model. The Exoglucanase (CBH) activity is assimilated to an erosion process as for soluble substrates. The $\beta$-glucosidase activity as well as the inhibition effect are also considered in the global population balance-based model. The numerical resolution of the population balance equation (PBE) is based on the Direct Quadrature Method of Moments (DQMOM) coupled with the Maximum Entropy (ME) reconstruction technique. Numerical results in different cases show coherent prediction of the particle size distribution (PSD) evolution during the hydrolysis reaction as well as the kinetics of simple sugars release. The modelling procedure is supported by experimental data which reveal the importance of the particles cohesion and its evolution during the hydrolysis reaction. Keywords: Enzymes, hydrolysis, population balance, particulate substrate, cohesion force
\end{abstract}

*. Corresponding author. Address : INSA, LISBP, 135, avenue de Rangueil, F-31077 Toulouse, France. Tel. : +33 56155 9774; Fax : +33 561559760

Email address: jerome.morchain@insa-toulouse.fr (Jérôme Morchain) 


\section{Introduction}

The production of bio-ethanol and chemicals from lignocellulose-based materials has attracted extensive research over the last few decades. This growing interest on renewable energy sources and green chemistry is motivated by the environmental and socio-economic issues as well as the depletion of fossil fuels Huang

5 et al. 2011).

The fractionation of the biomass into its elementary constituents by means of hydrolytic enzymes is known as the most challenging step of the substrate transformation process, it directly controls the conversion rate/yield thus, significant efforts have been devoted to (i) the improvement of the substrate pretreatments through a better understanding of the physico-chemical properties of the biomass in order to enhance its digestibility (ii) the understanding of the enzymes hydrolysis elementary mechanisms for improving the hydrolytic potential of the cocktails and (iii) the elucidation and quantification of the factors affecting the enzymes performance and the substrate reactivity during the hydrolysis reaction. These three contributions are necessary before one can develop reliable models for process design at industrial scale.

A typical enzymatic hydrolysis reaction kinetic shows an initial rapid rate followed by a progressive slowdown leading to a partial substrate conversion even for long-time reaction and/or high enzymes loadings (Levine et al., 2010). This makes the process cost uncompetitive. The decreasing rate of the biomass transformation is attributed to a multitude of factors acting synergistically at different levels (Mansfield et al., 1999). Thus, the modelling of this process-step is particularly challenging especially when dealing with insoluble substrates which is mostly the case.

The enzymatic hydrolysis of particulate substrates is a heterogeneous multi-step reaction. The enzymes are first adsorbed onto the substrate surface by their Carbohydrate-Binding Module (CBM) before acting with the Catalytic Domain (CD) and hydrolyze the glycosidic bonds (Hilden \& Johansson, 2004). As described in the first part, Michaelis-Menten type kinetic models are widely used for the modelling of this reaction (Bansal 
et al. 2009). In that case, the complex substrate architecture is not taken into account and the only variable of interest is the susbtrate bulk concentration. In adsorption-based kinetic models, Langmuir isotherms are used to quantify the fraction of adsorbed cellulases onto the initial substrate (Kadam et al., 2004). As the substrate surface changes all along the reaction as well as other substrate structural features (e.g. crystallinity, accessibility), the cellulases adsorption kinetics are affected (Chauve, 2011). All these effects are often lumped into a single time-decreasing function (e.g. ratio, negative exponential function) Kadam et al. 2004, Neto et al. 2013) referred as the substrate reactivity $\left(R_{s}\right)$ which is introduced in the modelling to reflect the dynamic character of the process (Zheng et al., 2009). Such simplifications, in both Michaelis-Menten and adsorption type-kinetic models, affect the reliability of these models and thus their ability to predict the timeevolution of the substrate properties and its hydrolysability as reported by Sin et al. (2010). To overcome these issues, the structure of the model has to be rethought. It is certainly interesting to build a model in which the specific area of the substrate particles is described. This motivated, among other arguments, the choice of the population balance formalism.

In the first part of this study, a population balance approach has been developed for the modelling of the enzymatic degradation of cellulose polymer chains. The three main cellulolytic activities have been taken into account. The EG activity is assimilated to a random scission of the polymer chains while the $\mathrm{CBH}$ activity is modelled as an erosion process leading to the release of a specific product : cellobiose. The $\beta$-glucosidase activity is then incorporated into the population balance model by the means of a Michaelis-Menten type kinetic. The Direct Quadrature Method of Moments (DQMOM) (Marchisio \& Fox, 2005) was retained as the solution method for the population balance equation (PBE) due to its low computational cost and its ability to treat the case of multidimensional problems (i.e. when a particle has to be defined by more than one property). This method is coupled with the Maximum Entropy technique for the reconstruction of the chain length distributions (CLDs) from its moments (Lebaz et al., 2016). The numerical implementation of the 
model has been validated first against an analytical solution of the PBE before testing the different activities independently and in combination. In addition to the very low computational cost (few seconds using a basic computer station), the model is able to predict EG-CBH synergistic effect as well as the inhibition of the enzyme activities due to the accumulation of the end-products (simple sugars).

This second part is devoted to the specific case of particulate substrates. The general theoretical framework remains unchanged. The population balance model (PBM) is extended to complex systems by rethinking how the different enzymatic activities are acting and degrading the substrate at the particulate scale. In particular, one has to consider now that an enzymatic attack does not imply the particle breakage but rather affects its cohesion. The particle fragmentation is due to the hydrodynamic shear stress. After introducing the theoretical framework, numerical results are discussed in different cases and experimental data are given in order to support the model assumptions.

\section{Theoretical framework}

The population balance modelling is a common approach when dealing with particulate/dispersed systems undergoing space and/or phase transformation. In our case, cellulose particles are progressively transformed into simple sugars (cellobiose and glucose) by the cooperative action of the cellulases.

In the first part of this study, a population balance model is formulated and solved numerically in the case of pure soluble cellulose substrates. This second part focuses on insoluble/particulate substrates. Considering the particle volume $v$ as the internal coordinate since it is the conservative variable, the following PBE ${ }_{65}$ (equation (1D) governs the evolution of the particle size distribution (PSD) in the specific case of a pure homogeneous fragmentation process :

$$
\frac{\partial n^{\prime}(v, t)}{\partial t}=\int_{v}^{\infty} \beta^{\prime}\left(v, v^{\prime}\right) \Gamma^{\prime}\left(v^{\prime}\right) n^{\prime}\left(v^{\prime}, t\right) d v^{\prime}-\Gamma^{\prime}(v) n^{\prime}(v, t)
$$


where $n^{\prime}(v, t)$ is the volume-based number density function, $\Gamma^{\prime}(v)$ the breakage frequency for a particle of volume $v, \beta^{\prime}\left(v, v^{\prime}\right)$ is the daughter distribution function giving the probability to obtain a particle of volume $v$ from the breakup of a particle of volume $v^{\prime}$ with $v^{\prime}>v$. The first term on the right hand side of equation (1) accounts for the formation (birth) of particles with volume $v$ resulting from the breakage of larger particles $v^{\prime}$. The last term is the death term reflecting the loss of particles of volume $v$ because of their breakage.

Assuming that the particle volume is proportional to its size, $v \propto L^{3}$ (with $L$ the particle size), equation (1) can be expressed in terms of particle length-based number density function $n(L, t)$ as (Marchisio et al. 2003) :

$$
\frac{\partial n(L, t)}{\partial t}=\int_{L}^{\infty} \beta(L, \lambda) \Gamma(\lambda) n(\lambda, t) d \lambda-\Gamma(L) n(L, t)
$$

To solve equation (2), numerical methods can be used. As described in the first part of this study, the DQMOM approach based on the time-tracking of a finite number of the PSD moments is adopted for its low computational cost and its robustness. This is of critical importance for the extension of the model to multivariate systems and/or the coupling with hydrodynamic phenomena. We recall below the physical meaning of the moments notion.

The $k^{t h}$ order moment of the PSD is defined as :

$$
m_{k}(t)=\int_{0}^{\infty} L^{k} n(L, t) d L
$$

The moments provide the main properties of the PSD. The zeroth order moment, $m_{0}(t)$, represents the molar concentration of the cellulose particles since $n(L, t) d L$ is the number of particles per volume having sizes between $L$ and $L+d L$. The first order moment, $m_{1}(t)$, gives the total length of cellulose particles per volume unit. Average properties of the CLD can be derived from the moments such as the number-averaged particle size given by the ratio $m_{1}(t) / m_{0}(t)$ and the Sauter mean diameter $D_{32}=m_{3}(t) / m_{2}(t)$. 
By applying the moment transformation to equation (2), we obtain the final moment transport equation below (for more detail, one can refer to the first part) :

$$
(1-k) \sum_{i=1}^{N} L_{i}^{k} a_{i}+k \sum_{i=1}^{N} L_{i}^{k-1} b_{i}=\sum_{i=1}^{N} \bar{b}_{i}^{(k)} \Gamma_{i} w_{i}-\sum_{i=1}^{N} L_{i}^{k} \Gamma_{i} w_{i}
$$

where

$$
\left\{\begin{aligned}
a_{i} & =\frac{\partial w_{i}}{\partial t} \\
b_{i} & =\frac{\partial c_{i}}{\partial t} \\
c_{i} & =w_{i} L_{i} \\
\bar{b}_{i}^{(k)} & =\int_{0}^{\infty} L^{k} \beta\left(L, L_{i}\right) d L
\end{aligned}\right.
$$

$\left(L_{i}(t), w_{i}(t)\right)$ are the abscissas and weights of the $N$ Gaussian quadrature nodes. $\left(L_{i}(0), w_{i}(0)\right)$ can be computed using the Product-Difference (PD) algorithm (Gordon, 1968) or any other algorithm (John \& Thein 2012). Equation (4) is similar to that developed in the first part, the only difference resides in the formulation of the breakage parameters (daughter distribution function and frequency).

\subsection{Daughter distribution functions formulation}

We propose thas the evolution of the PSD during the enzymatic hydrolysis of a particulate substrate is governed by both the cooperative action of the enzymes and the mechanical stress acting on the particle due to the fluid motion.

The EG activity breaks up the glycosidic bonds of surface chains affecting by the way the cohesion of the particles and their mechanical properties. The combination of this activity and the hydrodynamic shear stress leads ultimately to the particles fragmentation. Thus, unlike the case of soluble substrate where the EG attacks produce new chains systematically, in the case of particulate substrate, the fragmentation process is complex and both the EG activity and the shear stress have to be taken into account. The CBH activity attacks the free chain-ends and produces cellobiose molecules. This activity can be seen as a surface erosion 
process. Starting from these considerations, the PSD evolution during the enzymatic hydrolysis process is modelled using the following PBE :

$$
\frac{\partial n(L, t)}{\partial t}=S_{L}^{B}(L, t)+S_{L}^{C B H}(L, t)
$$

with

$$
\left\{\begin{aligned}
S_{L}^{B}(L, t) & =\int_{L}^{\infty} \beta_{B}(L, \lambda) \Gamma_{B}(\lambda) n(\lambda, t) d \lambda-\Gamma_{B}(L) n(L, t) \\
S_{L}^{C B H}(L, t) & =\int_{L}^{\infty} \beta_{C B H}(L, \lambda) \Gamma_{C B H}(\lambda) n(\lambda, t) d \lambda-\Gamma_{C B H}(L) n(L, t)
\end{aligned}\right.
$$

where $\beta_{B}$ and $\beta_{C B H}$ are the daughter distribution functions, $\Gamma_{B}$ and $\Gamma_{C B H}$ the breakage frequencies for EG/stress and CBH respectively.

We discuss below the choice of the daughter distribution functions and the breakage frequencies in this specific case of particulate substrate.

\subsubsection{EG activity and particle breakage}

In the case of heterogeneous reactions, as discussed before, the fragmentation mechanism of the substrate particles is complex. The EG activity acts at a microscopic scale by cutting glycosidic bonds without leading necessarily to the breakage of the attacked particles. The structural features of the substrate particles and the hydrodynamic shear stress are to be taken into account when dealing with the PSD evolution during the hydrolysis reaction. With a mono-component substrate such as cellulose, one can consider that all the particles have the same structural characteristics (e.g. degree of crystallinity, porosity). Thus, breakage may occur at any position on the particle surface. In other words, after the binary breakage of a particle, all sizes are equally probable. This can be formulated by a uniform daughter distribution function as in equation (8) (Marchisio et al., 2003).

$$
\beta_{B}\left(\lambda^{3}, L^{3}\right)=2\left(3 L^{2} / \lambda^{3}\right)=6 L^{2} / \lambda^{3}
$$


The source term $S_{L}^{B}(L, t)$ in equation $(7)$ can be explicited as :

$$
S_{L}^{B}(L, t)=6 L^{2} \int_{L}^{\infty} \frac{1}{\lambda^{3}} \Gamma_{B}(\lambda) n(\lambda, t) d \lambda-\Gamma_{B}(L) n(L, t)
$$

\subsubsection{CBH activity : chain-end scission}

The $\mathrm{CBH}$ activity, even for insoluble substrates, can be considered as an erosion process leading to a specific product which is a cellobiose molecule. In this case, an initial particle of volume $v$ losses elementary particles of volume $v_{c}$ after $\mathrm{CBH}$ attacks (equation $10 \mathrm{p}$ ).

$$
P(v) \longrightarrow P\left(v-v_{c}\right)+P\left(v_{c}\right)
$$

Thus, $\beta_{C B H}$ in this specific case is set as an erosion distribution function. Equations 11 and 12 give the time evolution of the particulate system under $\mathrm{CBH}$ attacks : equation (11) refers to the time evolution of the PSD while equation 12 describes accumulation of cellobiose due to the $\mathrm{CBH}$ activity).

$$
\begin{gathered}
\frac{\partial n^{\prime}(v, t)}{\partial t}=\int_{v}^{\infty} \Gamma_{C B H}\left(v^{\prime}\right) \delta\left(v-\left(v^{\prime}-v_{c}\right)\right) n\left(v^{\prime}, t\right) d v^{\prime}-\Gamma_{C B H}(v) n(v, t) \\
\frac{\partial n_{c}^{\prime}(t)}{\partial t}=\int_{v}^{\infty} \Gamma_{C B H}\left(v^{\prime}\right) \delta\left(v-v_{c}\right) n\left(v^{\prime}, t\right) d v^{\prime}
\end{gathered}
$$

The moment transformation of equations (9) and (11) is given in equation (4) in its gereral form. Equation (12) is to be included to the PBE solved by DQMOM after an appropriate moment transformation (see Appendix $\mathrm{C}$ of the first part of the study for more details).

\subsection{Breakage frequencies formulation}

Once the daughter distribution functions are properly defined for the two processes considering particulate substrates, the most challenging part is the formulation of the breakage frequencies $\Gamma_{B}$ and $\Gamma_{C B H}$ especially the first one. 
For the $\mathrm{CBH}$ activity, whatever the nature of the substrate, a specific product is released after an effective attack. Thus, $\Gamma_{C B H}$ matches with the $\mathrm{CBH}$ hydrolysis rate as for soluble substrates. Unlike $\Gamma_{C B H}, \Gamma_{B}$ is function of the substrate nature/properties and the operating conditions. In the case of soluble substrate, the rate of breakage of the polymer chains defines the intrinsic activity of the EG. But with insoluble substrate, there is a strong dissociation between the cutting of a glycosidic bond and the particle volume/size evolution since the length-scales are different.

Substrate particle breakup phenomenon occurs when the hydrodynamic force $F_{H}$ acting on the particle surface becomes higher than the cohesion force $F_{p}$ of the particle itself. Thus, the ratio of the two forces $\frac{F_{H}}{F_{p}}$ is deterministic (Coufort \& Line, 2003). The hydrodynamic force $F_{H}$ is proportional to the particle surface and can be explicited in the case of spherical particles as (Douaire et al., 2011) :

$$
F_{H}=\frac{5}{2} \eta \cdot G \cdot L^{2}
$$

where $G$ is the shear rate $\left(s^{-1}\right)$ and $\eta$ the dynamic viscosity $\left(k g \cdot m^{-1} \cdot s^{-1}\right)$.

The shear rate can be expressed as (Camp \& Stein, 1943) :

$$
G=\sqrt{\frac{\epsilon}{\nu}}
$$

where $\nu$ is the kinematic viscosity $\left(m^{2} \cdot s^{-1}\right)$ and $\epsilon$ is the average turbulent energy dissipation rate $\left(W \cdot k g^{-1}\right)$ which can be approximated via equation 15 below :

$$
\epsilon=\frac{P}{\rho_{f} V}=\frac{N_{p} N_{s}^{3} D^{5}}{V}
$$

where $P$ is the power input $(W), \rho_{f}$ is the fluid density $\left(\mathrm{kg} \cdot \mathrm{m}^{3}\right), N_{p}$ is the impeller power number, $N_{s}$ the impeller speed $\left(r \cdot s^{-1}\right), D$ is the impeller diameter $(m)$ and $V$ is the tank volume $\left(m^{3}\right)$. 
Thus, the hydrodynamic force $F_{H}$ exerted on a particle of size $L$ is explicited as :

$$
F_{H}=\frac{5}{2} \rho_{f}\left(\frac{\nu N_{p} N_{s}^{3} D^{5}}{V}\right)^{\frac{1}{2}} L^{2}
$$

The particle cohesion force $F_{p}$ is related to its density and its size and quantifies the mimimum force required to break up the particle. We propose to model this force as :

$$
F_{P}=A \cdot \rho_{p} \cdot e^{-\theta \Gamma_{E G} \cdot t} \cdot L^{\alpha}, \quad \alpha \in[1,3]
$$

with $A$ a constant, $\rho_{p}$ is the density of the particles $\left(\mathrm{kg} \cdot \mathrm{m}^{3}\right), \theta$ is non dimensional parameter related

155

$$
\Gamma_{B}=\varphi \cdot \frac{F_{H}}{F_{p}}
$$

with $\varphi$ a constant.

The particles breakage frequency $\Gamma_{B}$ is proportional to the ratio of the hydrodynamic and the cohesion forces. If $F_{H}>F_{p}$, the ratio is larger than 1 and the breakage phenomenon occurs. If one replaces the two 
forces by their expressions (equations 16 and 117$)$, one obtains :

$$
\Gamma_{B}=\varphi \cdot \frac{\rho_{f}\left(\frac{\nu N_{p} N_{s}^{3} D^{5}}{V}\right)^{\frac{1}{2}} L^{2}}{A \cdot \rho_{p} \cdot e^{-\theta \Gamma_{E G} \cdot t} \cdot L^{\alpha}}=B(t) \cdot L^{2-\alpha}
$$

The expression of the breakage frequency in equation 19 is coherent with the previous works on the breakage processes induced by both the hydrodynamic shear stress and the cohesion of the particles especially in the case of flocculation process which has been fully studied using experimental and numerical approaches based mainly on the population balance formalism (Spicer \& Pratsinis, 1996, Nopens et al. 2002, Ding et al. time-dependency of $B(t)$. This coefficient conveys an information relative to the age of a particle in the system and its exposure to endoglucanases attacks.

\subsection{The EG and $C B H$ inhibition effect}

As in the case of soluble substrates, one has to take into account the inhibition effect since the cellulases adopted here is to introduce inhibition constants for both EG and $\mathrm{CBH}$ activities and to express the breakage frequencies of these two activities as functions of the cellobiose concentration (equations (20) and (21)). Thus, the breakage frequencies decrease with the accumulation of cellobiose.

$$
\begin{gathered}
\Gamma_{E G}=\Gamma_{E G_{0}} \frac{K_{E G}}{C_{c}(t)+K_{E G}} \\
\Gamma_{C B H}=\Gamma_{C B H_{0}} \frac{K_{C B H}}{C_{c}(t)+K_{C B H}}
\end{gathered}
$$

where $C_{c}(t)$ is the cellobiose concentration, $K_{E G}$ and $K_{C B H}$ are the inhibition constants for EG and CBH respectively, $\Gamma_{E G_{0}}$ and $\Gamma_{C B H_{0}}$ are the intrinsic EG and $\mathrm{CBH}$ activities respectively. 


\subsection{The $\beta$-glucosidase activity}

Michaelis-Menten type kinetic model is adopted for the modelling of the $\beta$-glucosidase activity which transforms cellobiose into glucose. A competitive inhibition by the accumulation of glucose is incorporated as shown in equation 22 .

$$
\frac{d C_{G}}{d t}=\frac{V_{m} C_{c}}{K_{m}\left(1+\frac{C_{G}}{K_{P}}\right)+C_{c}}
$$

where $C_{G}$ is the glucose concentration, $K_{m}$ and $V_{m}$ the Michaelis-Menten parameters, $K_{P}$ the inhibition constant for the $\beta$-glucosidase activity.

\subsection{Global problem formulation}

Equation (23) summarizes the system of equations solved simultaneously to track the PSD evolution and the accumulation of simple sugars. This system includes the fragmentation and the erosion processes in the

90 first PBE, the accumulation of cellobiose in the second PBE and finally the accumulation of glucose in the third Michaelis-Menten kinetic equation.

$$
\left\{\begin{aligned}
\frac{\partial n(L, t)}{\partial t} & =6 L^{2} \int_{L}^{\infty} \frac{1}{\lambda^{3}} \Gamma_{B}(\lambda) n(\lambda, t) d \lambda-\Gamma_{B}(L) n(L, t) \\
& +\int_{L}^{\infty} \Gamma_{C B H}(\lambda) \delta\left(L-\left(\lambda-L_{C}\right)\right) n(\lambda, t) d \lambda-\Gamma_{C B H}(L) n(L, t) \\
\frac{\partial C_{C}(t)}{\partial t} & =\int_{L}^{\infty} \Gamma_{C B H}(\lambda) \delta\left(L-L_{C}\right) n(\lambda, t) d \lambda-\frac{1}{2} \frac{d C_{G}(t)}{d t} \\
\frac{d C_{G}(t)}{d t} & =\frac{2 V_{m} C_{C}}{K_{m}\left(1+\frac{C_{G}}{K_{P}}\right)+C_{C}}
\end{aligned}\right.
$$

The numerical resolution of the system 23 is carried out using a DQMOM approach coupled with a reconstruction technique based on the maximum entropy (ME) theory. The ode 45 integrator of MATLAB is used on a work-station Intel ${ }^{\circledR}$ Core $^{\mathrm{TM}_{\mathrm{i}}}$-3740QM $\mathrm{CPU}$ with a clock speed of $2.7 \mathrm{GHz}$ and $16 \mathrm{~GB}$ of installed memory (RAM). 


\section{Experimental procedure}

\subsection{Materials}

Microcrystalline celluloses (Avicel PH-102 and Avicel PH-105) were kindly provided by FMC BioPolymer (USA). These are pure insoluble cellulose substrates with mean sizes of 90 and $20 \mu m$ respectively (data from

the supplier). They have been chosen because of their significantly different initial sizes and their comparable physico-chemical properties mainly the cristallinity, as reported in literature (Kleinebudde, 1997, Jumaa et al. 2000). The cellulase enzyme used in this study was a commercial Trichoderma reesei cellulase preparation (Celluclast 1.5L) from Sigma-Aldrich (Germany). The cocktail contains the main cellulase activities : EG, $\mathrm{CBH}$ and $\beta$-glucosidases.

\subsection{Enzymatic hydrolysis}

The hydrolysis of the substrates was carried out in $50 \mathrm{mM}$ sodium acetate buffer $(\mathrm{pH}=4.8)$ at $40{ }^{\circ} \mathrm{C}$. Sodium azide $\mathrm{NaN}_{3}(0.1 \%)$ was supplemented to inhibit microbial growth during the reactions. For all the experiments, a $500 \mathrm{~mL}$ glass tank with a water jacket was used. The rotational speed was fixed to $200 \mathrm{rpm}$ using a blade mixer ( 4 inclined blades, $5 \mathrm{~cm}$ diameter, $45^{\circ}$ angle). The substrate loadings were fixed to $1 \%$ ${ }_{210}(\mathrm{w} / \mathrm{v})$ and those of cellulases to $30 \mathrm{FPU} / \mathrm{g}$ substrate. Samples were withdrawn and boiled for $10 \mathrm{mn}$ to stop the enzymatic hydrolysis. The samples are, then, centrifuged at $2000 \mathrm{~g}$ for $10 \mathrm{mn}$. The supernatants were analysed to reach the simple sugar concentrations while the solid fraction was diluted for size distribution analysis as described hereafter.

The yield of the enzymatic conversion of Avicel PH-102 and Avicel PH-105 into simple sugars (cellobiose and glucose) was calculated as (Khodaverdi et al., 2012) :

$$
\text { Yield }(\%)=\frac{[\text { Glucose }(\mathrm{g} / \mathrm{L})+\text { Cellobiose }(\mathrm{g} / \mathrm{L}) \times 1.053] \times 0.9}{\text { Cellulose }(\mathrm{g} / \mathrm{L})} \times 100
$$




\subsection{Particle size distribution determination}

The PSDs evolution during the enzymatic hydrolysis reactions were determined through laser diffraction analyses using a Mastersizer 2000 Hydro, Malvern Instruments Ltd. This ex-situ technique is based on the conversion of the detected scattered light into a particle size distribution thanks to the Mie's theory thus, respect to the recommended obscuration rate. The results are given in terms of volume fraction histograms distributed following a geometric sequence with a first term $u_{0}=0.01 \mu m$ and a common ratio $r=1.1482$ (total number of classes $=100)$.

\subsection{Mechanical resistance of the substrate particles}

The mechanical resistance of the initial substrate particles was explored using an ultrasonic bath (Ultrasonic Cleaning Unit Elmasonic S 60 (H), Elma Schmidbauer GmbH, Germany) having an operating frequency of $37 \mathrm{kHz}$. Diluted solutions are prepared in bechers which are placed in the bath for 5 minutes. The ultrasound effect on the substrate particles is revealed by the PSD analysis (Mastersizer 2000 Hydro).

\subsection{Sugar analysis}

(HPLC) on an Ultimate 3000 Dionex separation system equipped with a BioRad Aminex HPX 87H affinity column and a refractive index detector (Thermo Scientific). The separation of product species was performed in $\mathrm{H}_{2} \mathrm{SO}_{4}(5 \mathrm{mM})$ solution at $40{ }^{\circ} \mathrm{C}$ and at a flow rate of $0.3 \mathrm{~mL} / \mathrm{min}$. 


\section{Results and discussion}

$$
n(L, 0))=\frac{1}{\sigma \sqrt{2 \pi}} e^{-\frac{(L-\mu)^{2}}{2 \sigma^{2}}}
$$

Numerically, the parameters of the initial distribution are fixed as $\mu=70 \mu \mathrm{m}$ and $\sigma=5 \mu \mathrm{m}$. We discuss hereafter the numerical results given by the PBM resolution considering the different cellulolytic activities and the particle fragmentation processes. For the sake of clarity and concision, we report only the main results in each case.

\subsubsection{CBH activity}

The $\mathrm{CBH}$ activity is assimilated to a surfacic erosion process leading to the detachment of elementary particles. Thus, the erosion frequency is set proportional to $L^{2}$. The complexity in the case of insoluble substrates is the large difference between the substrate particle size (micron scale) and the elementary particle size (cellobiose with a sub-nanometer scale). Thus, numerically this considerable gap in terms of size scales is unmanageable since it generates round-off errors. The idea here is to consider a large collection of cellobiose molecules as an elementary particle released because of the erosion process. Thus, an elementary particle is not produced with one $\mathrm{CBH}$ attack but it results from a multitude of attacks which are gathered to produce 250 a significant fragment volume in order to produce a fragment with a significant volume from a numerical point of view. In our case, the size of an elementary particle is set to $0.2 \mu \mathrm{m}$. This size is sufficiently small compared to the mean size of the substrate particles.

We give in the two first figures the results obtained considering the erosion process only. Figure 1 gives the time-evolution of the four first reduced moments of the initial PSD. The zeroth order moment giving 

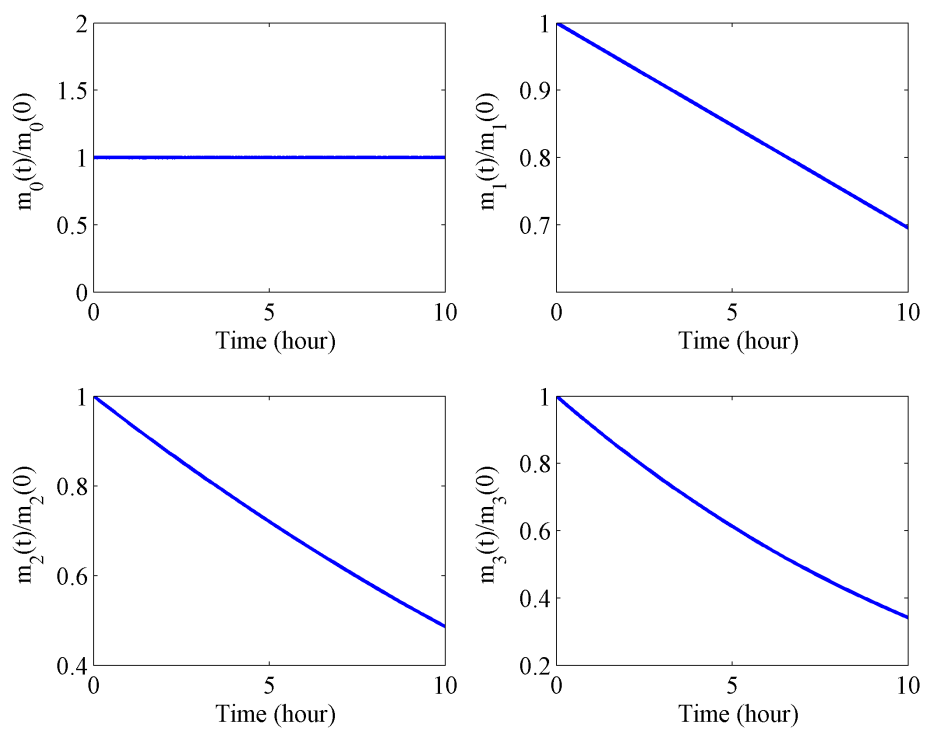

FiguRE 1: Time-evolution of the first four reduced moments : erosion process.

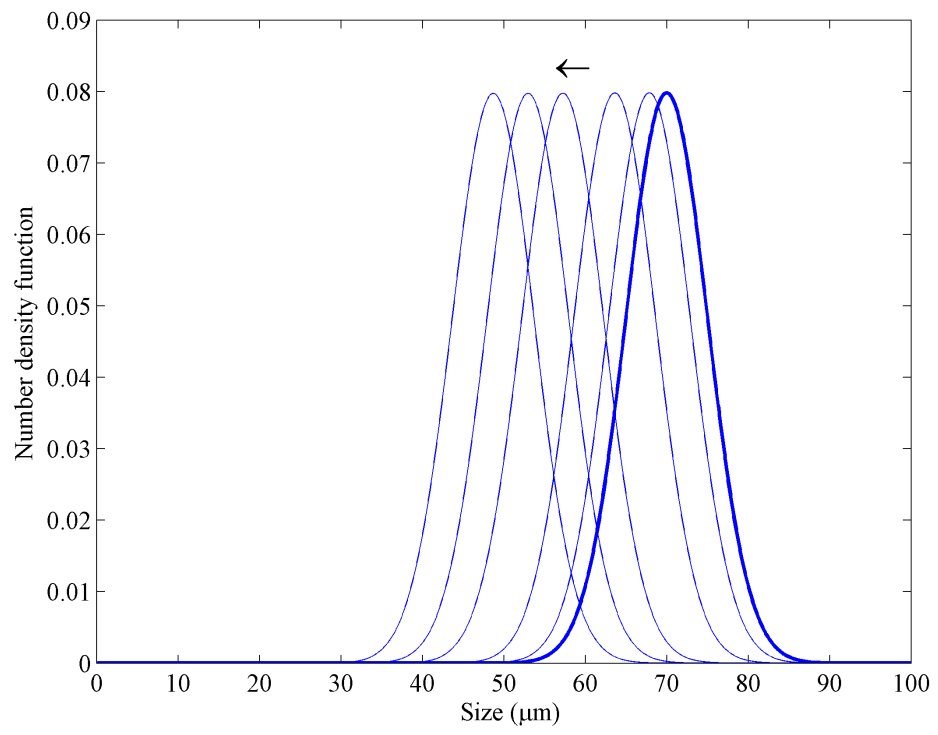

Figure 2: Time-evolution of the PSD undergoing CBH attacks. The PSDs from right to left correspond respectively to the times $t=0,1,3,6,8$ and 10 hours of hydrolysis.

the concentration of the substrate particles is constant since no new particles are formed in the system, the existing ones are becoming smaller thus, the first/second/third moments related respectively to the total length/surface/volume of the particles decrease continuously. Figure 2 shows the time-evolution of the initial 
PSD undergoing erosion process. The initial PSD expressed here as a number density function shifts toward the small sizes without any deformation since the concentration of the particles is kept unchanged, the decrease of the volume of the substrate particles is counterbalanced by the increase of the concentration of the simple sugars thus, the mass balance is satisfied.

\subsubsection{EG activity and fragmentation process}

For the particle fragmentation process, the breakage frequency is set proportional to the particle size $L$ which means that the factor $\alpha$ is set equal to one in equation $(19)$. Furthermore, to initialize the resolution procedure, the hydrodynamic force $F_{H}$ is estimated using the operating conditions explicited in the experimental procedure section. The cohesion force $F_{p}(t=0)$ satisfies the relation $\frac{F_{H}}{F_{p}(t=0)}<1$ which means that initially, the cohesion of the particles is greater than the hydrodynamic shear stress. In this case, the initial breakage frequency is minimal.
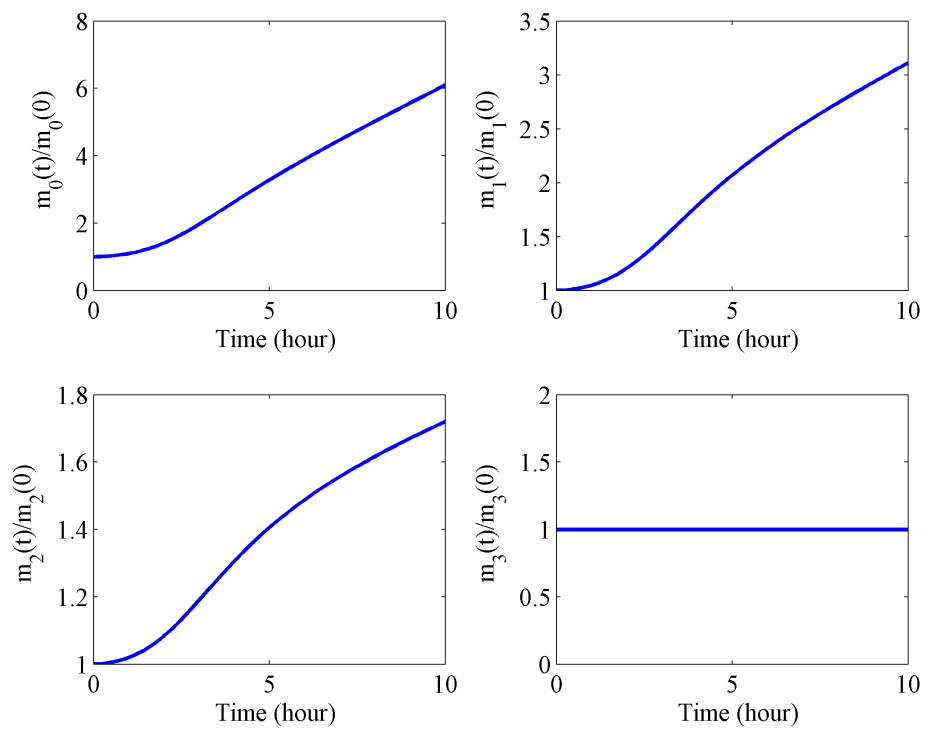

FiguRE 3: Time-evolution of the first four reduced moments : pure fragmentation process.

The aim of this study is not the accurate estimation of the cohesion force and its evolution within the 
distribution of fracture energies (Crespo, 2011, Frances \& Lin, 2014) have to be explored. Our purpose is to develop a modelling framework in which all these considerations can be easily taken into account and incorporated to a global simulation procedure.

Unlike the hydrodynamic force which is constant whithin the process time, the cohesion force decreases progressively since the substrate particles are attacked by the enzymes. We give an illustration of the fragmentation process in figures 3 and 4

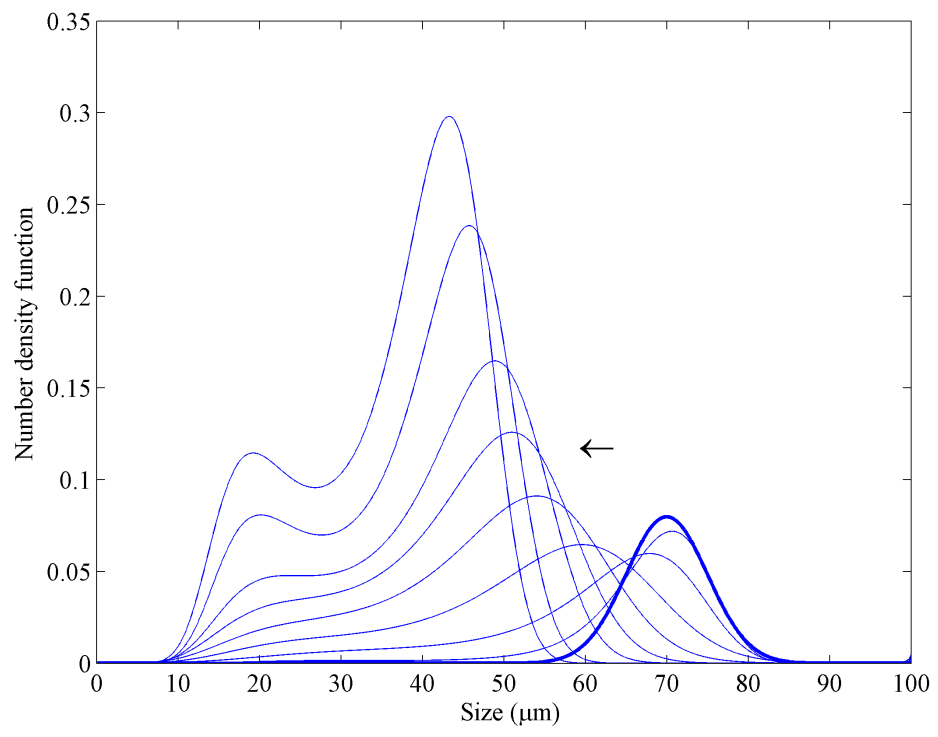

Figure 4: Time-evolution of the PSD undergoing a pure fragmentation process. The PSDs from right to left correspond respectively to the times $t=0,1,2,3,4,5,6,8$ and 10 hours of hydrolysis.

The evolutions of the first four reduced moments given in the Figure 3 show a slow initial rate as the cohesion force is important before an accelerating phase. The concentration of the particles increases considerably (zeroth order moment) as well as the total surface of the particles (second order moment). The total volume is kept unchanged (third order moment) since the fragmentation process is conservative.

The reconstruction of the PSDs from the moments using the ME technique in this case is illustrated in Figure 4. The initial PSD shifts toward the small sizes with a substantial shape deformation. Since the 
fragmentation frequency is proportional to the particle size, the rate of transformation of the largest particles is high compared to that of small ones. fragmentation frequency proportional to the particle size $(L)$. The substrate fraction converted into cellobiose by the $\mathrm{CBH}$ activity is further transformed into glucose by the $\beta$-glucosidase activity. The inhibition effect is introduced for the three different activities. The results are illustrated in the figures $5,6,77$ and 8
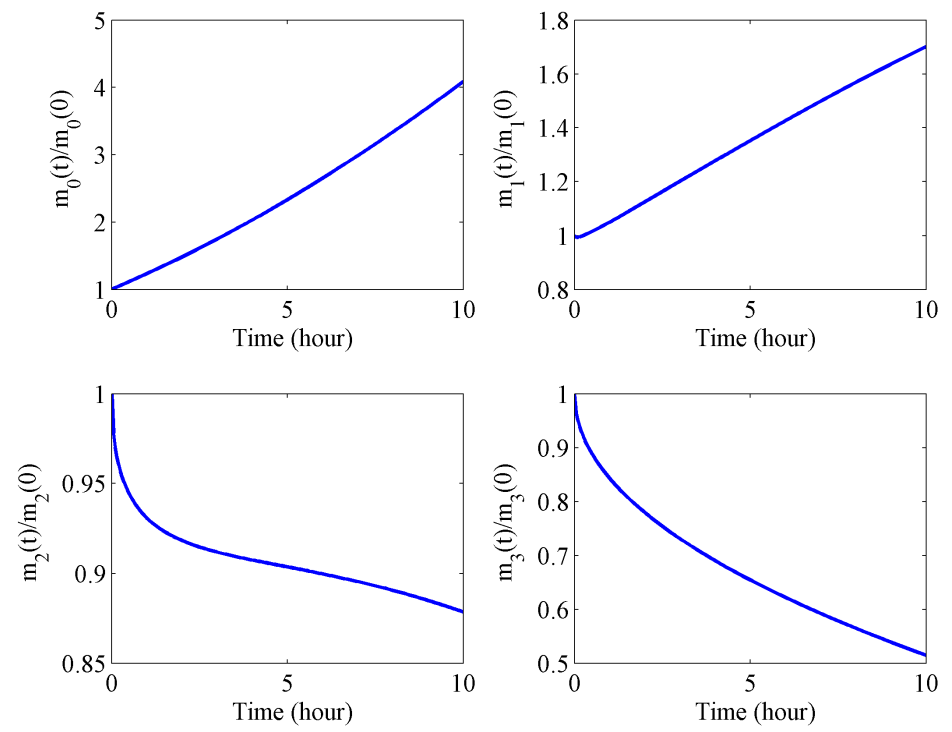

Figure 5: Time-evolution of the first four reduced moments : pure fragmentation and erosion process simultaneously. 


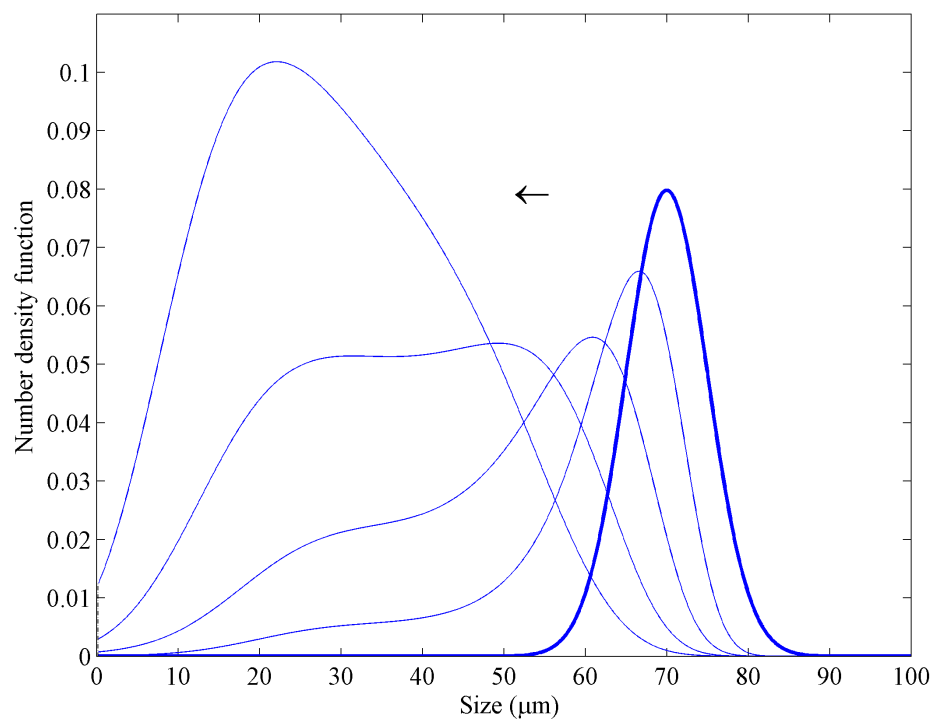

Figure 6: Time-evolution of the PSD undergoing pure fragmentation and erosion processes. The PSDs from right to left correspond respectively to the times $t=0,1,3,6$ and 10 hours of hydrolysis.

The accumulation of the small particles formed after the disintegration of the large ones is becoming more and more important. The ME reconstruction method is very interesting here because $n(0, t)$ and $\left.\frac{\partial n(L, t)}{\partial L}\right|_{0}$ are not constrained to be zero.

Since the conservative variable in the case of particulate substrates is the particle volume, we give in Figure 7 the evolution of the volume based density function during hydrolysis reaction derived from the evolution of the number density function (Figure 6) assuming the sphericity of the particles. Recall that this assumption has been formulated in the theoretical framework section in order to transform the PBE written in terms of volume-based density function into number density function based PBE. As we can see in Figure 7. a tail toward the small sizes is formed as a consequence of the fragmentation process and the integral of the distribution decreases in time because of the erosion process.

The conversion of the substrate into simple sugars (Figure 8) shows a rapid initial phase followed by a progressive decreasing rate step. The slower production rate is due to the inhibition effect : the accumulation 


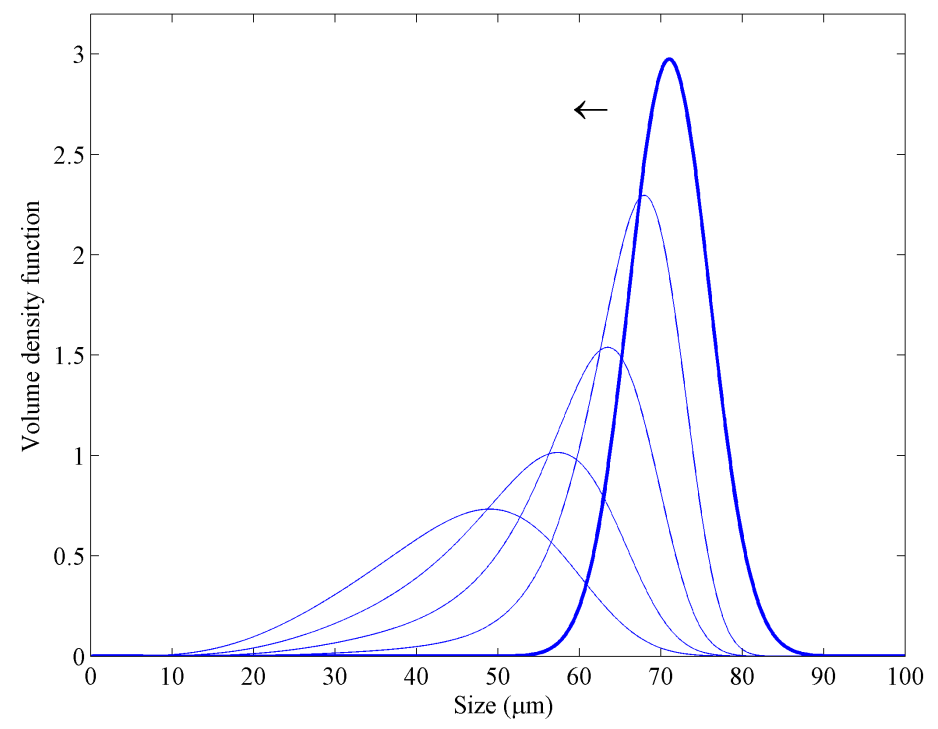

Figure 7: Time-evolution of the volume-based density distribution undergoing pure fragmentation and erosion processes. The distributions correspond from right to left respectively to the times $t=0,1,3,6$ and 10 hours of hydrolysis.

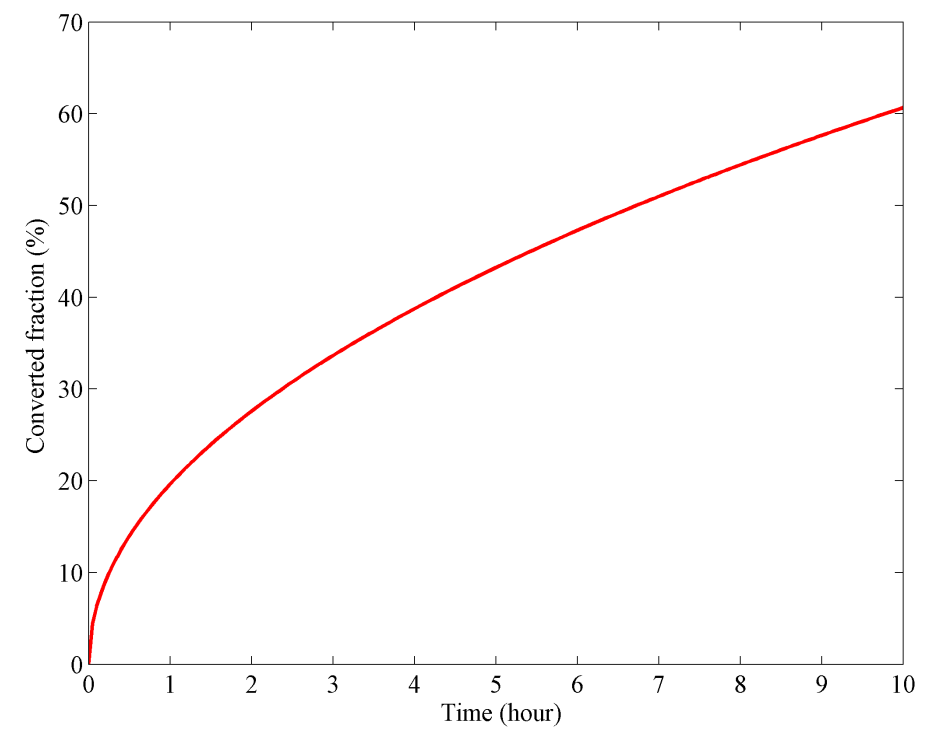

FIGURE 8: Evolution of the converted fraction of the substrate into simple sugars in the case of EG-CBH activities with the inhibition effect

of the simple sugars affects negatively the enzyme activities. This typical profile is in agreement with the experimental results as it will be discussed in the next section. 
In this section, we give some experimental results obtained using particulate cellulose (Avicel PH-102 and Avicel PH-105) submitted to enzymatic hydrolysis under the conditions commonly used in literature. The experiments described below aim to support our modelling approach such as the importance of the substrate structure and the evolution of the physical properties of the substrates during the hydrolysis reaction. The population balance framework developed in this contribution is based on these considerations which is not the case for Michaelis-Menten based kinetic models widely used in literature.

\subsubsection{Mechanical properties of the substrates}

The two different substrates used in this study (Avicel PH-102 and Avicel PH-105) have different initial PSDs. Solutions from these substrates are prepared with the same loading $(1 \% \mathrm{w} / \mathrm{v})$ and sonicated during 5 mn. The PSDs before and after sonication are measured using the laser diffraction analyser for each substrate.

The results are given in figures 9 and 10 .

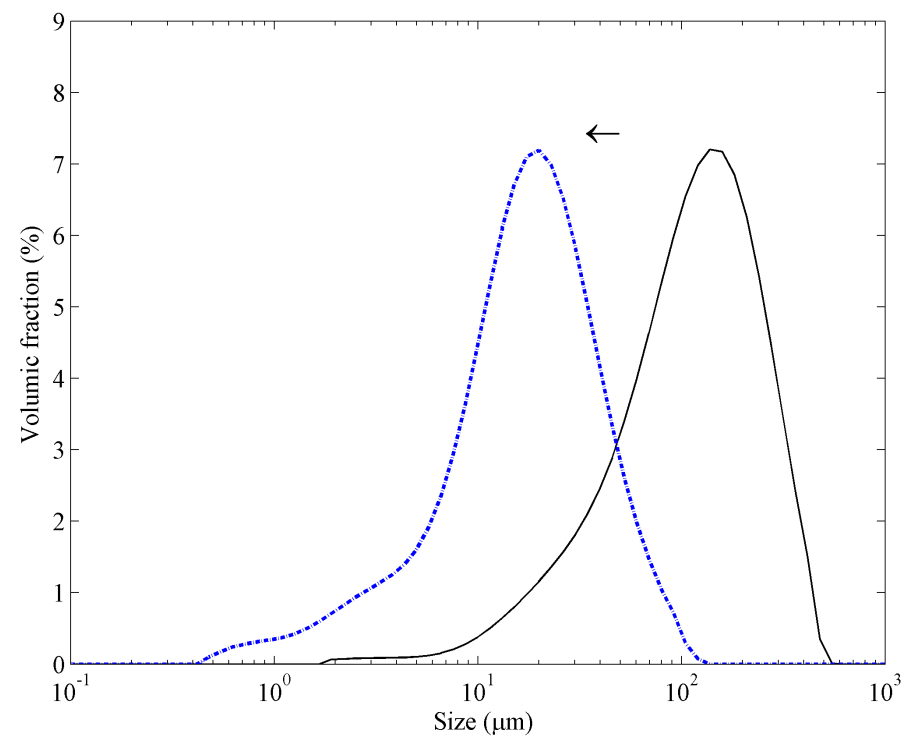

FIGURE 9: Ultrasound effect on the particle size distribution of Avicel PH-102 determined using laser diffraction analysis : Initial PSD (continuous line) and PSD after sonication (dashed line). 


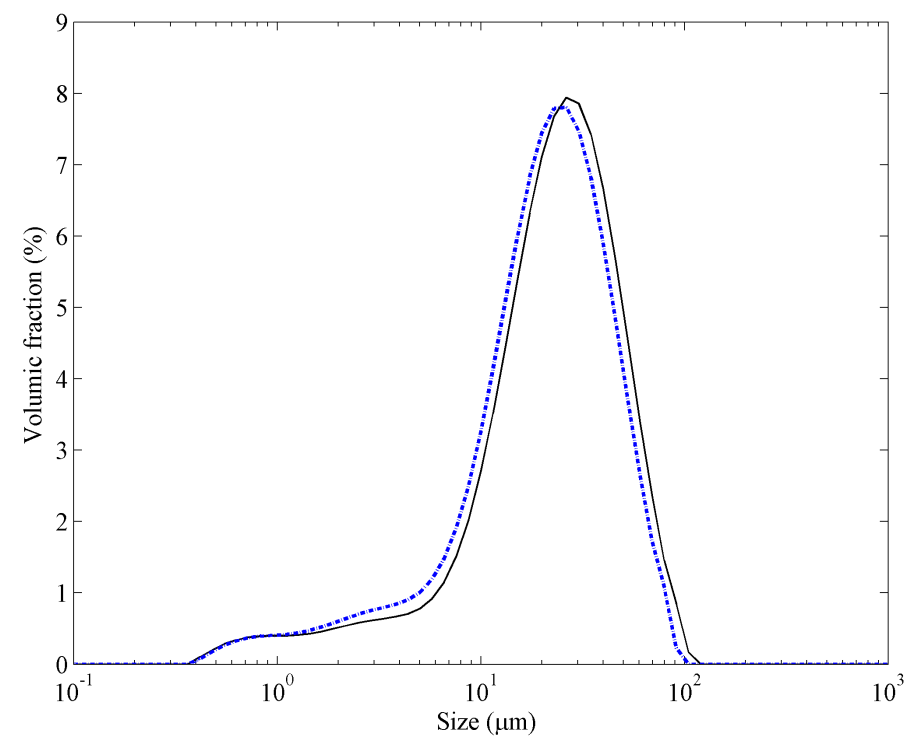

Figure 10: Ultrasound effect on the particle size distribution of Avicel PH-105 determined using laser diffraction analysis : Initial PSD (continuous line) and PSD after sonication (dashed line).

The figures 9 and 10 reveal a substantial different behaviour of the two substrates after sonication. The PSD of Avicel PH-102 initially centered around a mean size of $D_{43}\left(t_{i}\right)=140 \mu \mathrm{m}$ shifts completely toward small sizes so that, after 5 minutes of sonication, the new PSD is centered around $D_{43}\left(t_{f}\right)=22 \mu \mathrm{m}$ (Figure 9). The energy dissipated by the sonic waves is sufficient to affect the cohesion of the particles and leads ultimately to their disintegration into smaller particles. The behaviour of Avicel PH-105 is definitely different. The PSD is hardly affected by the sonication (Figure 10), its mean size changes from $D_{43}\left(t_{i}\right)=30 \mu \mathrm{m}$ to $D_{43}\left(t_{f}\right)=28 \mu \mathrm{m}$. This gives an indication on the cohesion force of the substrate particles which is sufficiently high to face the sonication energy which is not the case for Avicel PH-102.

This simple test shows the importance of the cohesion force of the substrate particles when dealing with particulate systems. Thus, eventhough the two substrates are pure cellulose with comparative crystallinity index, their mechanical resistance is substantially different. This means that their behaviour during the enzymatic hydrolysis reaction will be also completely different since the cohesion of the substrate particles 
will be distinctly affected by the hydrodynamic shear stress and the enzyme activities. This will be confirmed

\subsubsection{Enzymatic hydrolysis of Avicel PH-102 and Avicel PH-105}

The enzymatic hydrolyis reactions have been conducted for 24 hours using the two different substrates under the same conditions. The evolution of the PSDs as well as the conversion into simple sugars have been tracked all along the reactions. The results are given in the figures 11,12 and 13 .

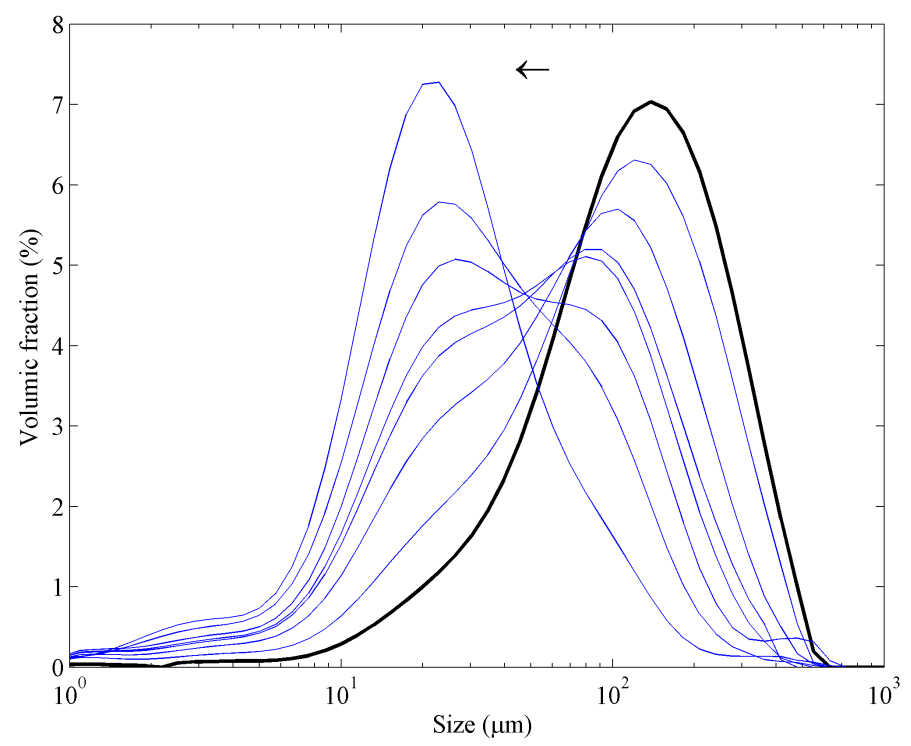

Figure 11: Particle size distribution evolution during the enzymatic hydrolysis of Avicel PH-102. The distributions from right to left correspond respectively to the times $t=0,1,2,3,4,6,8$ and 24 hours of hydrolysis.

As expected, the PSD of Avicel PH-102 shows a spectacular evolution. After 24 hours of hydrolysis, the final PSD is completely different from the initial one. The mean size of the substrate particles moves from $D_{43}\left(t_{i}\right)=140 \mu \mathrm{m}$ to $D_{43}\left(t_{f}\right)=33 \mu \mathrm{m}$. The total converted fraction in this time interval is around $25 \%$.

In the case of Avicel PH-105, the evolution of the PSD is not significant compared to that of Avicel PH102. Indeed, the mean size of the substrate particles decreases from $D_{43}\left(t_{i}\right)=30 \mu \mathrm{m}$ to just $D_{43}\left(t_{i}\right)=24 \mu \mathrm{m}$ in 24 hours of hydrolysis (Figure 12). However the kinetic of simple sugars release follows exactely that of Avicel PH-102 as shown in Figure13. The examination of the Figure 12 shows that with the slight translation 


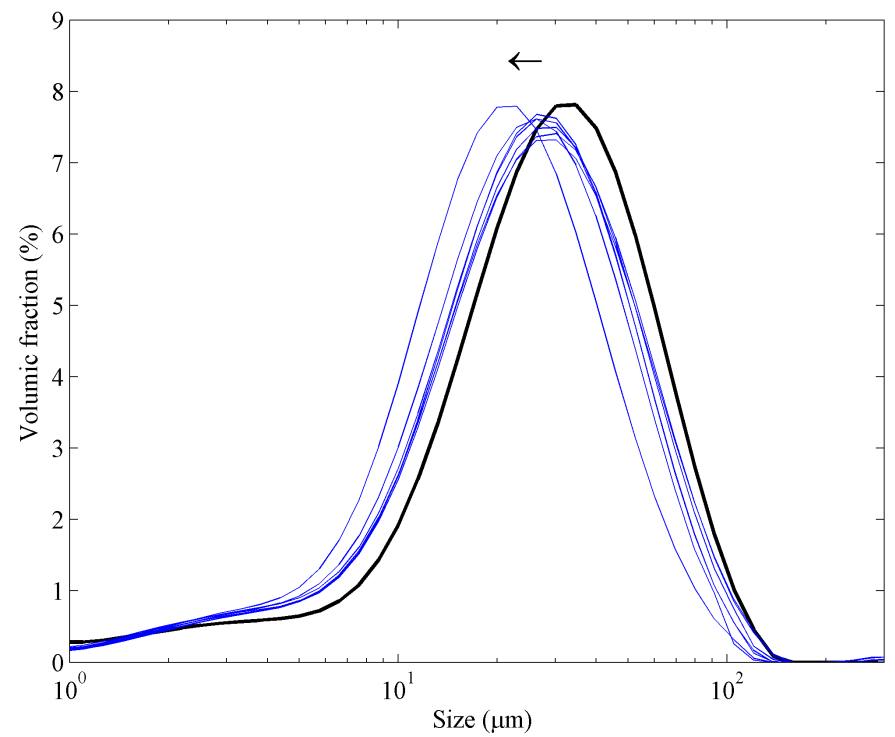

Figure 12: Particle size distribution evolution during the enzymatic hydrolysis of Avicel PH-105. The distributions from right to left correspond respectively to the times $t=0,1,2,3,4,6,8$ and 24 hours of hydrolysis.

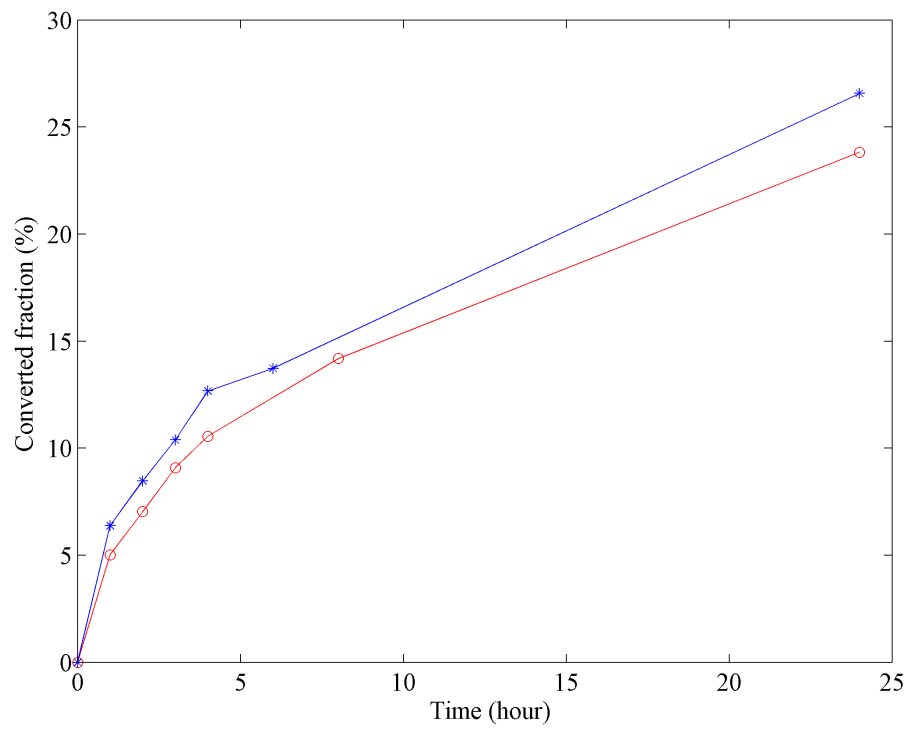

Figure 13: Exprimental substrate converted fraction evolution during enzymatic hydrolysis of Avicel PH-102 (red circles) and Avicel PH-105 (blue stars).

of the initial PSD into small sizes, the global shape is preserved. This can be explained by the high cohesion force of the substrate particles thus, the erosion process is dominating. 
The results of the enzymatic hydrolysis reactions corroborate those discussed in the previous section where

of having access to the PSD evolution in parallel to the conversion rate to be able to interpret the results of hydrolysis reactions appropriately. 


\section{Conclusion and perspectives}

initially for the soluble substrates has been extended to insoluble substrates since these systems are ubiquitous when dealing with enzymatic hydrolysis reactions.

The transition to the particulate substrates brings additional challenges since the particle fragmentation process is dissociated from the intrinsic cellulolytic activities which was not the case when dealing with chain induces automatically the breakup of this chain and the formation of two smaller ones. In the case of an insoluble cellulose particle, the action of the EG activity leads to the breakage of an accessible glycosidic bond without the fragmentation of the particle. This activity affects the structural features of the substrate particles mainly their cohesion force. Thus, the fragmentation process has been modelled by taking into is still considered as an erosion process since it leads to a specific product even for particulate substrates. The novelty here is the formulation of the daughter distribution function which has been rethought since the length scales are extremely distinct between the cellobiose molecules and the substrate particles. The $\beta$-glucosidase activity is modelled as a michaelian kinetic and the inhibition effect has been incorporated the Direct Quadrature Method of Moments (DQMOM) coupled with the Maximum Entropy reconstruction technique.

The numerical tests in different cases show a consistent results for both the fragmentation and the erosion processes. The population balance model predicts the evolution of the substrate initial PSD and the conversion

properties of the cellulose particles. The two different substrates tested present distinct behaviours against this 
assay. Avicel PH-102 is extremely sensitive to the ultrasounds which induce a severe fragmentation process while Avicel PH-105 is minimally affected. The enzymatic hydrolysis reactions conducted with identical conditions show the same results. The particle size distribution of Avicel PH-102 evolves along the reaction which is poorly the case of that of Avicel PH-105 but the conversion rates are similar. These experimental tests support the modelling approach and point to the importance of the coupling between the PSD evolution and the conversion rate for a judicious interpretation of the hydrolysis reaction results.

The dynamic character of the cohesion force of the substrate particles which can be characterised by the notion of the age of the substrate intervenes in our case in the formulation of the breakage frequency of the substrate particles. The introduction of this notion as a new variable leading to a multivariable population balance model would be of great importance for process considerations. This allows the monitoring of the sequencial addition of the substrate for example. Finally, the added value of the modelling approach presented in this contribution is its possible extension for multivariable systems without losing its robustness.

\section{Aknowledgments}




\section{Nomenclature}

A Constant appearing in the cohesion force

$B(t) \quad$ Parameter of the breakage frequency

$a_{i} \quad$ First time derivative of the weights of the quadrature nodes

$b_{i} \quad$ First time derivative of the product of the weighted abscissas

$C_{C} \quad$ Cellobiose molar concentration

$C_{G} \quad$ Glucose molar concentration

$c_{i} \quad$ Weighted abscissas

D Impeller diameter

$F_{H} \quad$ Hydrodynamic force

$F_{p} \quad$ Particle cohesion force

G Shear rate

$k \quad$ Moments order

$K_{C B H} \quad$ Inhibition constant of the $\mathrm{CBH}$

$K_{E G} \quad$ Inhibition constant of the EG

$K_{m} \quad$ Parameter of the Michaelis-Menten type kinetic

$K_{P} \quad$ Inhibition constant of the $\beta$-glucosidase

$L \quad$ Particle size

$L_{i} \quad$ Abscissas of the quadrature nodes

$m_{k}(t) \quad k^{t h}$ order moment

$n(L, t) \quad$ Number based particle size distribution

$n^{\prime}(v, t) \quad$ Volume based particle size distribution

$N \quad$ Number of quadrature nodes

$N_{p} \quad$ Impeller power number

$N_{s} \quad$ Impeller speed

$P \quad$ Dissipated power

$S_{L}^{B}(L, t) \quad$ Source term of the PBE accounting for the pure breakage process

$S_{L}^{C B H}(L, t) \quad$ Source term of the PBE accounting for the erosion process

$t \quad$ Time

$v \quad$ Particle volume

V Tank volume

$v_{c} \quad$ Volume of the elementary particle for the erosion process

$V_{m} \quad$ Parameter of the Michaelis-Menten type kinetic

$w_{i} \quad$ Weights of the quadrature nodes 


\section{Greek Symbols}

$\begin{array}{ll}\alpha & \text { Exponent } \\ \beta_{B} & \text { Daughter distribution function for the breakage process } \\ \beta_{C B H} & \text { Daughter distribution function for the erosion process } \\ \delta\left(v-v_{c}\right) & \text { Dirac delta function centered on } v_{c} \\ \epsilon & \text { Average turbulent energy dissipation rate } \\ \varphi & \text { Cefficient of the breakage frequency } \\ \Gamma_{B} & \text { Particle breakage frequency } \\ \Gamma_{C B H} & \text { Erosion frequency for CBH activity } \\ \Gamma_{C B H_{0}} & \text { Intrinsic erosion frequency for CBH activity } \\ \Gamma_{E G} & \text { Chains breakage frequency for EG activity } \\ \Gamma_{E G_{0}} & \text { Intrinsic chains breakage frequency for EG activity } \\ \lambda & \text { Particle size } \\ \mu & \text { Mean of the PSD } \\ \nu & \text { Kinematic viscosity } \\ \eta & \text { Dynamic viscosity } \\ \rho_{f} & \text { Volumic mass of the fluid } \\ \rho_{p} & \text { Volumic mass of the substrate particles } \\ \sigma & \text { Standard deviation } \\ \theta & \text { Coefficient of particle's fatigue }\end{array}$

\section{Abreviations}

$\mathrm{CBH} \quad$ Cellobiohydrolase

CBM Carbon-Binding Module

CD Catalytic Domain

CLD Chain Length Distribution

DQMOM Direct Quadrature Method of Moments

EG Endoglucanase

FPU Filter Paper Unit

HPLC High Performance Liquid Chromatography

ME Maximum Entropy

PBE Population Balance Equation

PD Product-Difference algorithm

$\mathrm{pH} \quad$ Potentail of hydrogen

PSD Particle Size Distribution

Rs Substrate reactivity 


\section{References}

Bansal, P., Hall, M., Realff, M. J., Lee, J. H., \& Bommarius, A. S. (2009). Modeling cellulase kinetics on lignocellulosic substrates. Biotechnology Advances, 27, 833-848. URL : http://www.sciencedirect.com/ science/article/pii/S0734975009001402, doi $: 10.1016 /$ j.biotechadv.2009.06.005

Camp, T. R., \& Stein, P. C. (1943). Velocity gradients and internal work in fluid motion. Journal of the Boston Society of Civil Engineers, 85, 219-37. URL : http://bases.bireme.br/cgi-bin/wxislind.

Chauve, M. (2011). Modlisation cintique de l'hydrolyse enzymatique des substrats cellulosiques. Ph.D. thesis INSA, Toulouse. URL : http://www.theses.fr/2011GRENV066/abes

Coufort, C., \& Line, A. (2003). Forces on spherical particles in terms of upstream flow characteristics. 口 Chemical Engineering Research and Design, 81, 1206-1211. URL : http://www.sciencedirect.com/

Crespo, E. F. (2011). Application of particle fracture energy distributions to ball milling kinetics.

口 Powder Technology, 210, 281-287. URL : http://wWw.sciencedirect.com/science/article/pii/ S0032591011001458,

Ding, A., Hounslow, M. J., \& Biggs, C. A. (2006). Population balance modelling of activated sludge 
tococcus lactis cultivated in a Couette bioreactor. Biotechnology and bioengineering, 108, 559-571. URL : com/doi/10.1002/aic.14358/full

Gordon, R. G. (1968). Error bounds in equilibrium statistical mechanics. Journal of Mathematical Physics, http://onlinelibrary.wiley.com/doi/10.1002/bit.22974/full

Frances, C., \& Lin, A. (2014). Comminution process modeling based on the monovariate and bivariate direct quadrature method of moments. AIChE Journal, 60, 1621-1631. URL : http://onlinelibrary.wiley.

9, 655-663. URL : http://scitation.aip.org/content/aip/journal/jmp/9/5/10.1063/1.1664624.

Hilden, L., \& Johansson, G. (2004). Recent developments on cellulases and carbohydrate-binding modules ․ with cellulose affinity. Biotechnology letters, 26, 1683-1693. URL : http://link.springer.com/article/ $10.1007 / \mathrm{s} 10529-004-4579-8$

Huang, R., Su, R., Qi, W., \& He, Z. (2011). Bioconversion of Lignocellulose into Bioethanol : Process Intensification and Mechanism Research. BioEnergy Research, 4, 225-245. URL : http://link.springer. com/article/10.1007/s12155-011-9125-7, doi :10.1007/s12155-011-9125-7.

John, V., \& Thein, F. (2012). On the efficiency and robustness of the core routine of the quadrature method of moments (QMOM). Chemical Engineering Science, 75, 327-333. URL : http://www.sciencedirect. com/science/article/pii/S000925091200187X. the properties of extruded pellets. Part I. Physicochemical characterisation of the cellulose types after homogenisation. Colloid and Polymer Science, 278, 597-607. URL : http://link.springer.com/article/

\subsection{7/s003960000292.}

Kadam, K. L., Rydholm, E. C., \& McMillan, J. D. (2004). Development and validation of a kinetic model 

http://onlinelibrary.wiley.com/doi/10.1021/bp034316x/full.

Khodaverdi, M., Jeihanipour, A., Karimi, K., \& Taherzadeh, M. J. (2012). Kinetic modeling of rapid enzymatic hydrolysis of crystalline cellulose after pretreatment by NMMO. Journal of industrial microbiology $\&$ biotechnology, 39, 429-438. URL : http://link.springer.com/article/10.1007/s10295-011-1048-y. and spheronization. Pharmaceutical research, 14, 804-809. URL : http://link.springer.com/article/

\subsection{3/A:1012166809583.}

Lebaz, N., Cockx, A., Sprandio, M., \& Morchain, J. (2016). Reconstruction of a distribution from a finite number of its moments : A comparative study in the case of depolymerization process. Computers S0098135415003038 doi $10.1016 / j \cdot$ compchemeng.2015.09.008.

Levine, S. E., Fox, J. M., Blanch, H. W., \& Clark, D. S. (2010). A mechanistic model of the enzymatic 口 hydrolysis of cellulose. Biotechnology and bioengineering, 107, 37-51. URL : http://onlinelibrary. wiley.com/doi/10.1002/bit.22789/full. cellulose hydrolysis. Biotechnology progress, 15, 804-816. URL : http://onlinelibrary.wiley.com/ doi/10.1021/bp9900864/full.

Marchisio, D. L., \& Fox, R. O. (2005). Solution of population balance equations using the direct quadrature method of moments. Journal of Aerosol Science, 36, 43-73. URL : http://www.sciencedirect.com/ 
Marchisio, D. L., Pikturna, J. T., Fox, R. O., Vigil, R. D., \& Barresi, A. A. (2003). Quadrature me-

q thod of moments for population-balance equations. AIChE Journal, 49, 1266-1276. URL : http:

//onlinelibrary.wiley.com/doi/10.1002/aic.690490517/abstract.

Neto, J. M., dos Reis Garcia, D., Rueda, S. M. G., \& da Costa, A. C. (2013). Study of kinetic parameters in a mechanistic model for enzymatic hydrolysis of sugarcane bagasse subjected to different pretreatments.

Bioprocess and biosystems engineering, 36, 1579-1590. URL : http://link.springer.com/article/10. $1007 / \mathrm{s} 00449-013-0930-6$

Nopens, I., Biggs, C. A., De Clercq, B., Govoreanu, R., Wilen, B. M., Lant, P., \& Vanrolleghem, P. A. (2002). Modelling the activated sludge flocculation process combining laser light diffraction particle sizing and population balance modelling(PBM). Water Science \& Technology, 45, 41-49. URL : http://modeleau. fsg.ulaval.ca/fileadmin/modeleau/documents/Publications/pvr317.pdf.

Sin, G., Meyer, A. S., \& Gernaey, K. V. (2010). Assessing reliability of cellulose hydrolysis models to support biofuel process designidentifiability and uncertainty analysis. Computers 83 chemical engineering, 34 , 1385-1392. URL : http://www.sciencedirect.com/science/article/pii/S0098135410000554. distribution. AIChE Journal, 42, 1612-1620. URL : http://onlinelibrary.wiley.com/doi/10.1002/ aic.690420612/full,

Xiao, Z., Zhang, X., Gregg, D. J., \& Saddler, J. N. (2004). Effects of sugar inhibition on cellulases and -glucosidase during enzymatic hydrolysis of softwood substrates. In Proceedings of the Twenty-Fifth Symposium on Biotechnology for Fuels and Chemicals Held May 47, 2003, in Breckenridge, CO (pp. 1115-1126).

Springer. URL : http://link.springer.com/chapter/10.1007/978-1-59259-837-3_90. 
Zheng, Y., Pan, Z., Zhang, R., \& Jenkins, B. M. (2009). Kinetic modeling for enzymatic hydrolysis of \. pretreated creeping wild ryegrass. Biotechnology and bioengineering, 102, 1558-1569. URL : http:// onlinelibrary.wiley.com/doi/10.1002/bit.22197/full 